\title{
Signals for dynamical process from IMF-IMF correlation function
}

\author{
E.V. Pagano*1, L. Acosta ${ }^{2,3}$, L. Auditore ${ }^{2,4}$, T. Cap $^{5}$, G. Cardella ${ }^{2}$, M. Colonna ${ }^{1}$, E. De \\ Filippo $^{2}$, E. Geraci ${ }^{2,6}$, B. Gnoffo ${ }^{2,6}$, G. Lanzalone ${ }^{1,7}$, C. Maiolino ${ }^{1}$, N. Martorana ${ }^{1,6}$, A.

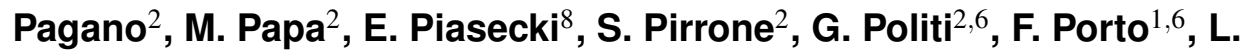 \\ Quattrocchi $^{2,6}$, F. Rizzo ${ }^{1,6}$, P. Russotto ${ }^{1}$, A. Trifiro' ${ }^{2,4}$, M. Trimarchi ${ }^{2,4}$ and K. \\ Siwek-Wilczynska ${ }^{9}$ \\ ${ }^{1}$ INFN Laboratori Nazionali del Sud, Catania, Italy \\ ${ }^{2}$ INFN Sezione di Catania, Catania, Italy \\ ${ }^{3}$ Instituto de Fisica, Universidad Nacional Autonoma de Mexico, Mexico \\ ${ }^{4}$ Dipartimento di Scienze MIFT, Universita' Messina, Italy \\ ${ }^{5}$ National Centre for Nuclear Research, Otwock-Swierk, Poland \\ ${ }^{6}$ Dipartimento di Fisica e Astronomia, Universita' di Catania, Italy \\ ${ }^{7}$ Universita' di Enna "Kore", Enna, Italy \\ ${ }^{8}$ Heavy Ion Laboratory, University of Warsaw, Poland \\ ${ }^{9}$ Faculty of Physics, University of Warsaw, Poland \\ E-mail: epaganodlns.infn.it
}

\begin{abstract}
In this paper we briefly discuss about a novel application of the IMF- IMF correlation function in the physics case of heavy projectile-like (PLF) binary fission-like massive splitting in heavy ion collisions at Fermi energy. In particular, results are shown for the reverse kinematics reaction ${ }^{124} \mathrm{Sn}+{ }^{64} \mathrm{Ni}$ at $35 \mathrm{AMeV}$ that has been investigated by using the forward part of CHIMERA multi-detector. Transport CoMD simulations are compared with the data. The main analysis was performed as a function of the charge asymmetry of the decay channel. The coexistence of dynamical and statistical components as a function of the charge asymmetry of the binary breakup is clearly evidenced. We show that the IMF-IMF correlation functions, when coupled with transport calculations, can be used to pin down the timescale of the fragments production and consequently the relevant ingredients of the in medium effective interaction. Efforts are being planned to extend the method to different reaction mechanisms and nuclear systems and, also, to compare the data with different transport theory simulations.
\end{abstract}

55th International Winter Meeting on Nuclear Physics

23-27 January, 2017

Bormio, Italy

\footnotetext{
* Speaker.
} 


\section{Introduction}

In a nuclear reaction at Fermi energies two and multi particles intensity interferometry correlation methods are useful tools in order to extract space-time information about their emission process [1]. For instance, space-time characteristics about light particle (like protons) emission sources are commonly understood in the frame of the Koonin-Pratt equation [2] using correlation functions under the condition of small relative linear momentum [3]. Evidently, for Intermediate Mass Fragments (IMF) correlations, larger relative momenta are expected due to the strong influence of the long range Coulomb repulsion. However, also for IMFs correlation functions, analysis gives valuable and unique information about the reaction mechanism and time scale [4-9]. The coexistence of dynamical and statistical mechanism in the emission of intermediate mass fragments (IMFs) is an important aspect of the reaction at Fermi energy that is characterised by a strong competition between mean field and nucleon-nucleon dynamics where in-medium particle interactions and the asymmetry term of the nuclear equation of state (ASyEOS) are important ingredients. First studies of correlation methods were done in the past for central and ternary neck emissions events with CHIMERA data $[10,11]$. In this paper we present a preliminary work where the IMF-IMF correlation function is applied to a well-known physics case previously studied by the CHIMERA collaboration, that is the dynamical fission of the projectile-like-fragment (PLF) in ${ }^{124} \mathrm{Sn}+{ }^{64} \mathrm{Ni}$ system at $35 \mathrm{~A} \mathrm{MeV}$ reverse reaction [12,13]. The basic idea is to provide a time-scale calibration of the IMF-IMF correlation function in that specific case and to study its shape evolution as a function of the fission-like mass asymmetry. The substantial difference of our method with respect to the well-known particle-particle femtoscopy studies [14-16], comes from the fact that in our physical case the detected pairs of particles (IMFs from PLF fission) are associated with high relative momenta as large as the strong relative exit channel IMF-IMF Coulomb repulsions. In this case, as in similar cases, a rather consistent way to extract both space-time and reaction mechanism information is to compare the experimental data with simulations including Coulomb trajectories calculations. In this work we present comparisons with the CoMD transport model $[17,18]$

\section{Experimental analysis}

In the present paper, we deal with IMFs particles defined as having atomic number between 3 and 25. To select projectile-like fission-like decay, we restrict our analysis to events with IMFs multiplicity equal to two. In agreement with previous studies $[12,13]$, a constraint on the IMFs velocity, requiring that both of the two fragments had the parallel velocity with respect to the beam axis greater than $5 \mathrm{~cm} / \mathrm{ns}$ was implemented. Notice that in the studied system the velocity of the centre of mass in the laboratory frame is $\approx 5.2 \mathrm{~cm} / \mathrm{ns}$ and the projectile velocity is $\approx 8$ $\mathrm{cm} / \mathrm{ns}$. As it is common in CHIMERA analysis, complete events were characterized by a total detected parallel momentum greater than $60 \%$ of the projectile one and, in our case, the total collected atomic number Ztot was required to be greater than 40 . As in previous analysis $[9,10]$, the charge asymmetry ratio $Z_{A s y}=Z_{H} / Z_{L}$ of the emitted pairs of IMFs, where $Z_{H}$ and $Z_{L}$ are the atomic numbers of the heavy and the light IMF of the pair, respectively, is adopted to classify and characterize the exit channel decay properties. So in order to characterize space-time properties of the IMF-IMF correlation function, the selected set of IMFs was decomposed in three partitions as a 
function of the following asymmetry ratios: $1 \leq Z_{A s y} \leq 2,2<Z_{A s y} \leq 4$ and $Z_{A s y}>4$. Furthermore, to select IMFs coming from the PLF source, an additional severe restriction was required in the sum of the charges of the two IMFs, i.e., $25 \leq Z_{H}+Z_{L} \leq 50$. As it is common, the experimental correlation function

$$
1+R\left(\left|\mathbf{V}_{\text {red }}\right|\right)=\mathbf{C}_{\mathbf{1 2}} \frac{\mathbf{Y}_{\text {Coinc }}\left(\left|\mathbf{V}_{\text {red }}\right|\right)}{\mathbf{Y}_{\text {Uncor }}\left(\left|\mathbf{V}_{\text {red }}\right|\right)}
$$

was evaluated by the ratio between coincident collected pairs of IMFs (in the same event) and uncorrelated ones. The denominator $Y_{\text {Uncor }}\left(\left|\mathbf{V}_{\text {red }}\right|\right)$ was computed with the event mixing technique [19]. This technique could be affected by the presence of some "non-femtoscopic" effects at small relative momenta [20,21], mostly related with the class of peripheral collisions that suggest some cautions when the correlation function is used as experimental reference for time scale and size evaluations under the assumption of typical femtoscopy equations [2, 15, 22]. However, in our study event mixing computations have been carefully compared with different techniques, as suggested in [19] such as, pseudo singles computations and Montecarlo pseudo-random samplings mapping the available phase space configuration. However, as stressed above, our analysis is not based on femtoscopy equations like in Refs [2, 15,22], but was performed by comparisons with transport simulations and the simulated events have been treated with the same procedures applied to the experimental data.

The three IMF-IMF correlation functions, as a function of the three ranges of the $Z_{A s y}$, are shown in Fig. 1 and they have been evaluated as a function of the reduced velocity defined as it follows [6]:

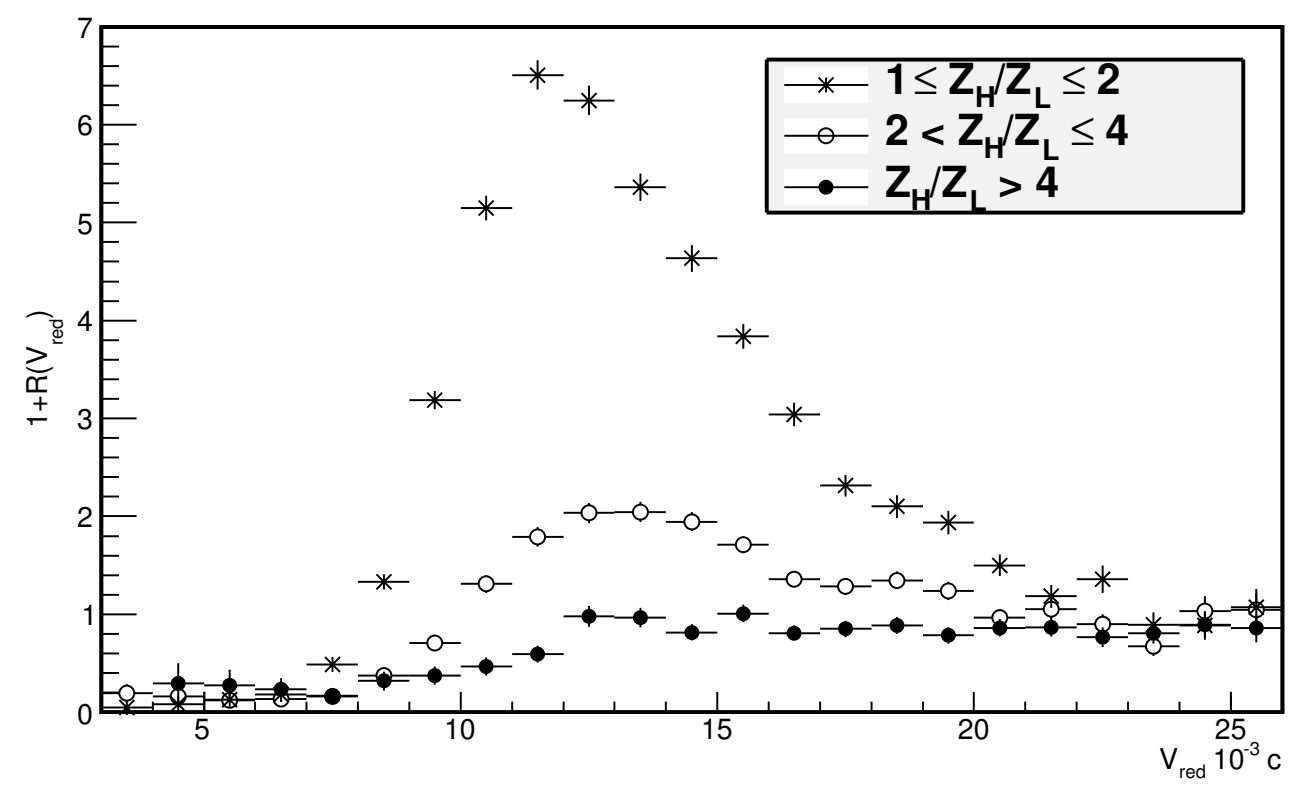

Figure 1: IMF-IMF correlation functions for $25 \leq Z_{H}+Z_{L} \leq 50$ gated by different $Z_{A s y}$ conditions [4]. 


$$
\mathbf{V}_{\text {red }}=\frac{\mathbf{V}_{\mathbf{H}}-\mathbf{V}_{\mathbf{L}}}{\sqrt{\mathbf{Z}_{\mathbf{H}}+\mathbf{Z}_{\mathbf{L}}}}=\frac{\mathbf{V}_{\text {rel }}}{\sqrt{\mathbf{Z}_{\mathbf{H}}+\mathbf{Z}_{\mathbf{L}}}}
$$

This quantity allows to add together pairs of particles having different atomic numbers [6] in order to increase the statistics that plays a crucial role in such studies. The IMF-IMF correlation functions, for the three ranges of charge asymmetry show very different shapes. It is worth to notice that the well-defined bump observed for $1 \leq Z_{A s y} \leq 2$ is centred at a value of reduced relative velocity that is in agreement with a binary sequential PLF splitting, Ãz deduced by the Coulomb repulsion as given by the Viola systematics [23]. The energy dissipation is analysed by inspecting the charged particle multiplicity in the three ranges of charge asymmetry (left panel of Fig. 2) and the corresponding kinematics properties of the sum $Z_{H}+Z_{L}$, (right panel of Fig. 2). On the left panel it is clearly evidenced the tendency of the dissipation to grow up with the increase of the asymmetry from a most probable value around 7 charged particles, for the most symmetric gate, up to about 10 charged particles for the most asymmetric one. This tendency is also confirmed by inspecting the right panel of Fig. 2 where it is clearly noticed that the most probable value of the sum of the atomic number of IMFs pairs consistently falls down with the increase of the $Z_{A s y}$. The $Z_{\text {Asy }}$ of the decay has a nexus with the energy dissipation of the collision path, and it consequent influences the "size" of the emitting system (PLF) of the correlated pairs.
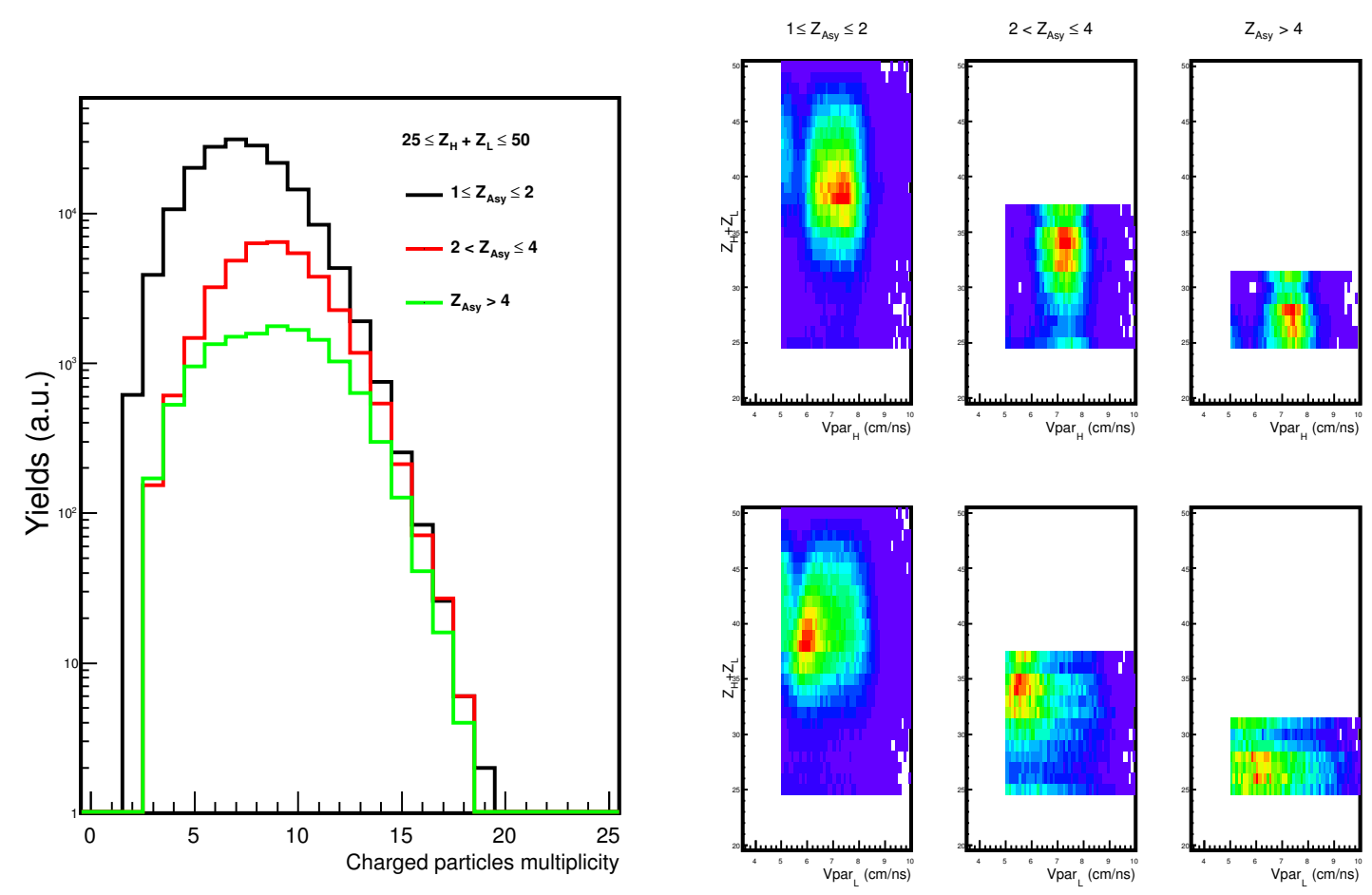

Figure 2: left panel: total charged particles multiplicity for the three ranges of $Z_{A s y}$ charge asymmetry. Right panel: $Z_{H}+Z_{L}$ as a function of the parallel velocity of the Heavy IMF (upper) or as a function of the parallel velocity of the Light one (bottom).

Assuming that the sum $Z_{H}+Z_{L}$ is a measure of the size of the emitting source, it is argued 
that the shape evolution of the correlation function shown in Fig. 1 is, at this stage, very poorly constrained with respect to both dissipation and size of the sources. So, in order to achieve a more careful understanding of the different shapes observed as a function of the charge asymmetry, it was mandatory to improve the class of the selected events with respect to the size of the emitting system and (consequently) the range of the energy dissipation. We have considered two complementary narrower bins of the sum of the atomic number of the two pairs, i.e., one bin defined in the range, $25 \leq Z_{H}+Z_{L} \leq 35$ (left panel of Fig. 3) and the other one in the range, $35 \leq Z_{H}+Z_{L} \leq 50$ (right panel of Fig. 3). According to data of the Fig. 3 in each one of the two bins the size of the source is reasonably fixed and practically the different charge asymmetries experience the same charged particles multiplicity distribution and, consequently, the same energy dissipation (assuming, as a crude approximation, that the multiplicity is a reasonable indicator of the dissipation). In Fig. 4 the new results for the IMF-IMF correlation functions are shown: in the left panel for the most dissipative range $25 \leq Z_{H}+Z_{L} \leq 35$ and in the right panel for the less dissipative $35 \leq Z_{H}+Z_{L} \leq$ 50 one. In the first case (larger dissipation) hierarchies in both intensity and shape as a function of the $Z_{A s y}$ are preserved; in contrast, in the second case (lower dissipation) the correlation functions show no significant differences as a function of the $Z_{A s y}$, within the error bars (notice that the larger asymmetry is not shown because in this case the statistics was too low). Our interpretation is that in the most dissipative range the changing shape of the correlation function is a clear indication of the evolutionary time scale character of the nuclear mechanism leading to the production of the IMFs. Instead, for the most gentle collisions associated with sizes in the range $35 \leq Z_{H}+Z_{L} \leq 50$ the space-time configuration is only poorly affected by the asymmetry parameter (practically, both shape and intensity of the correlation functions are unchanged).

Once the size of the emitting source is reasonable fixed by the value of $25 \leq Z_{H}+Z_{L} \leq 35$, the evolution of the intensity and shape of the correlation function with respect to the charge asymmetry is linked to the time scale of the decay process from the prompt dynamical decay (fast process) up to the equilibrated fission decay (sequential process). However, in order to achieve a better understanding of this time evolution, the correlation functions are studied as a function of the theta proximity angle $\theta_{\text {prox }}$, which is a powerful and independent indicator of both equilibrium emission and dynamical emission processes (if any) in the physics case under study [24]. In brief, the theta proximity angle tells us about the angular distribution of a selected light IMF of the pair with respect to a well reconstructed centre of mass vector velocity of the emitting PLF (see ref. [24] for more details).

In left panel of the Fig. 5 the distribution of the $\cos \left(\theta_{\text {prox }}\right)$ for the full range $25 \leq Z_{H}+Z_{L} \leq$ 50 and for the most symmetric gate of charge asymmetry is shown. In a pure sequential fission decay such a distribution is expected to be forward-backward symmetric with respect to the value of $\cos \left(\theta_{\text {prox }}\right)=0$. According with the previous careful analysis of refs. $[12,13]$ the distribution of Fig. 5 (left panel) is dominated by equilibrated sequential PLF fission decay and only a small but sizeable part $(\approx 10 \%)$ of it is associated with a prompt dynamical process evidenced by a forward angular asymmetry for the events with a $\cos \left(\theta_{\text {prox }}\right)$ close to 1 . In this peculiar case of small contribution of the dynamical process, it is very interesting to test the sensitivity of the shape of the correlation function to the $\cos \left(\theta_{\text {prox }}\right)$ forward-backward asymmetry. To do this, in the right panel of Fig. 5 three correlation functions have been evaluated with and without constraints in $\cos \left(\theta_{\text {prox }}\right)$ distribution. All the three correlations are peaked at nearly the same value of the reduced rela- 

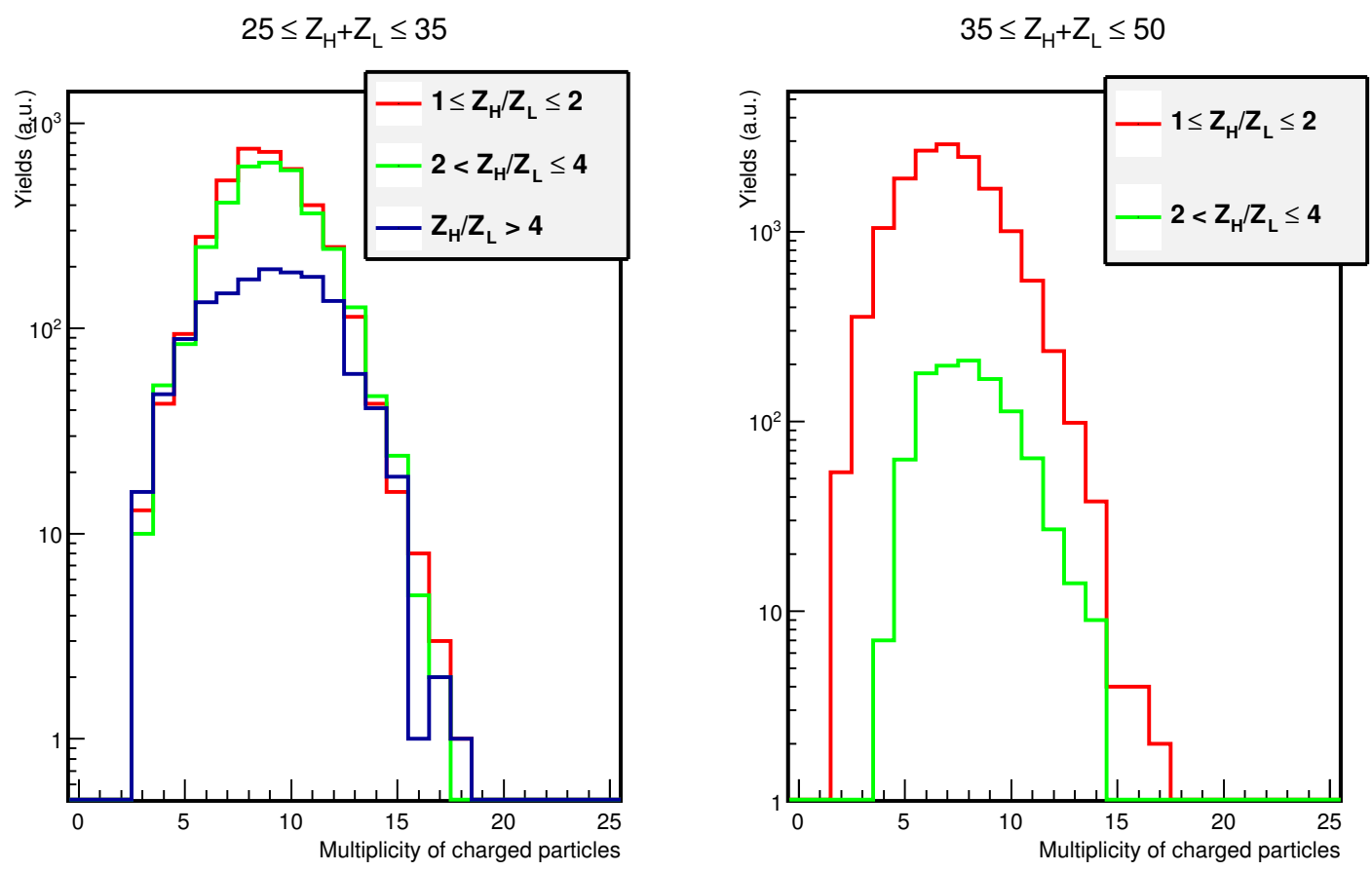

Figure 3: Total charged particles multiplicity as a function of $Z_{A s y}$, for the two bins of $25 \leq Z_{H}+Z_{L} \leq 35$ (left panel) and $35 \leq Z_{H}+Z_{L} \leq 50$ (right panel) [4].
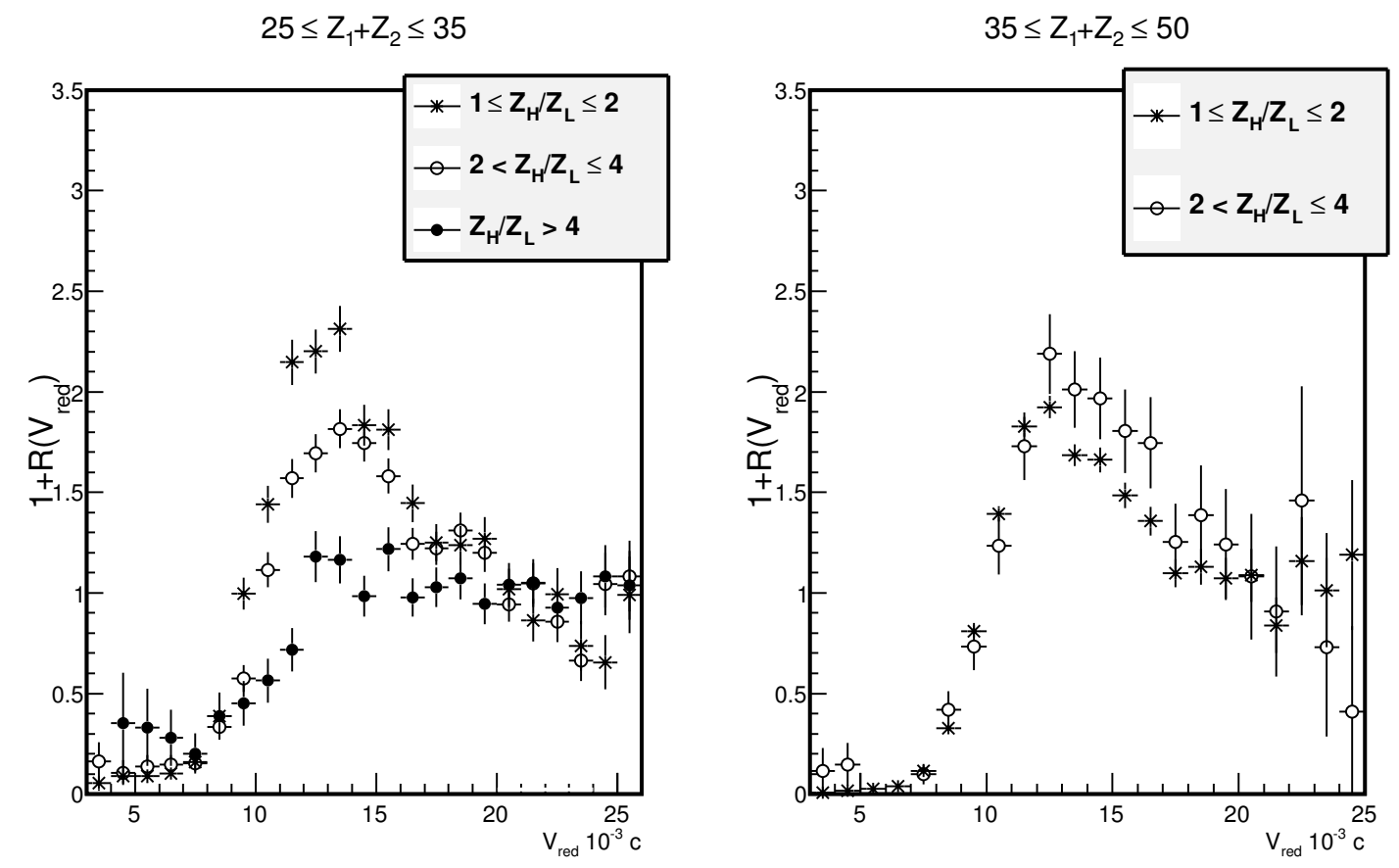

Figure 4: IMF-IMF correlation function for different $Z_{A s y}: 25 \leq Z_{H}+Z_{L} \leq 35$ (left panel) and $35 \leq$ $Z_{H}+Z_{L} \leq$ (right panel) [4]. 
$1 \leq Z_{H} / Z_{L} \leq 2,25 \leq Z_{H}+Z_{L} \leq 50$

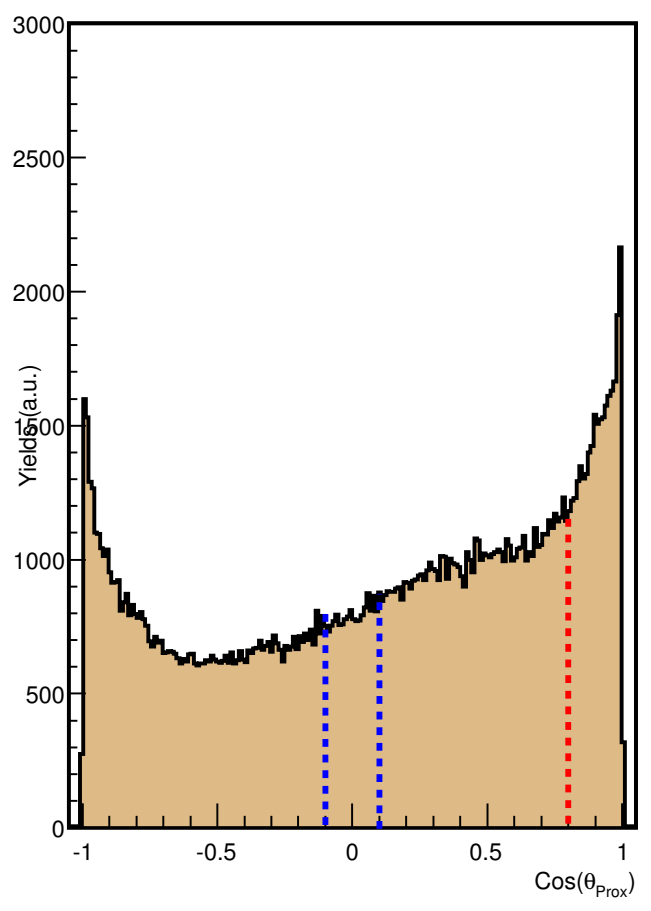

$1 \leq Z_{H} / Z_{L} \leq 2,25 \leq Z_{H}+Z_{L} \leq 50$

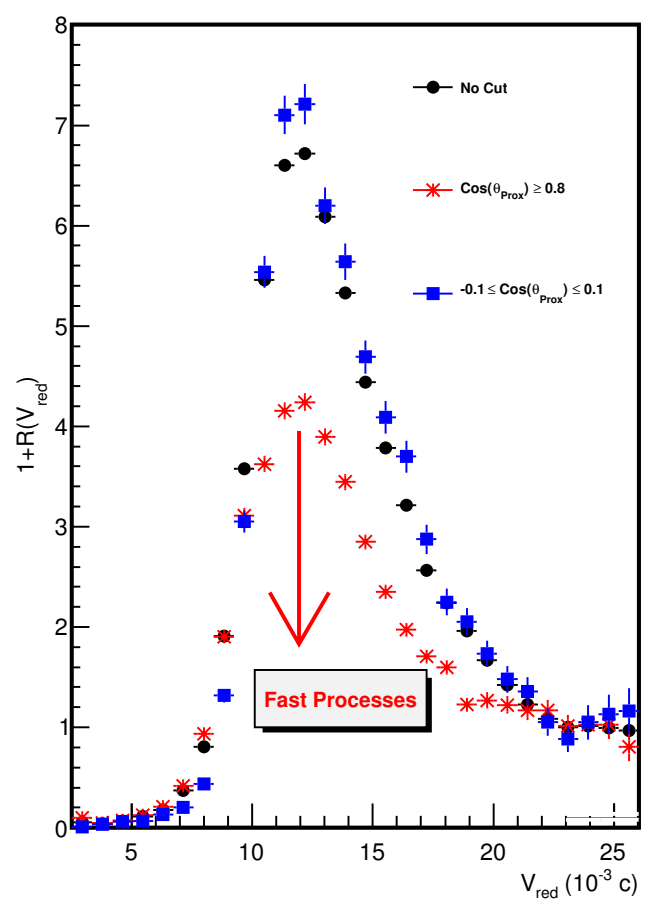

Figure 5: (left panel) distribution of the cosines of $\theta_{\text {prox }}$ for $25 \leq Z_{H}+Z_{L} \leq 50$ and $1 \leq Z_{A s y} \leq 2$. (Right panel) IMF correlation functions with and without constrains in $\cos \left(\theta_{\text {prox }}\right)$.

tive velocity (see Fig. 1 and text). The correlation function with the largest correlated intensity (blue-full square) corresponds to the one obtained constraining the $\cos \left(\theta_{\text {prox }}\right)$ (bin of 0.2 width) in the range of values $-0.1 \leq \cos \left(\theta_{\text {prox }}\right) \leq 0.1$ where a pure statistical fission-like sequential decay was evidenced $[12,13]$ and it is identical (within the accuracy) to the one (black-full dots) corresponding to the total $\cos \left(\theta_{\text {prox }}\right)$ distribution. The third correlation function (red-stars) is calculated constraining the total $\cos \left(\theta_{\text {prox }}\right)$ distribution for values (bin of 0.2 in width as in the black full dots one) $\cos \left(\theta_{\text {prox }}\right) \geq 0.8$, in the region where the strong forward-backward asymmetry shows the largest contribution of a dynamical-out of equilibrium fission-like process. It is clearly noticed that the intensity of this latter correlation function is strongly decreased and its shape is broadened; this is a further strong and complementary indication of the high sensitivity of the correlation function to sizeable contributions of the prompt/dynamical processes.

\section{Transport simulations and comparisons}

In order to pin down quantitative information about the timescale of the dynamical process we made comparisons between the experimental data and the CoMD model calculations $[17,18]$. In Fig. 6, e.g., for charge asymmetry in the range, $1 \leq Z_{A s y} \leq 2$, detailed comparisons between the main experimental observables ( a): $Z_{H}+Z_{L} ;$ b): $Z_{A s y}$ and c): $\cos \left(\theta_{\text {prox }}\right)$ distributions) used in 
this analysis and the ones calculated by the simulations with CoMDIII - EOS parametrization [18], are shown. In Fig. 6, beside CoMDIII simulation, CoMDII simulation parametrization [17] is also shown as alredy used in the preliminary analisys of refs [4,5], where sequential decays of the primary fragments were also incluted. Noticed that small discrepances between CoMDIII simulations and experimental data shown in Fig. 6 ( a), b) and c) ) are consistent with the fact that sequential decay is not included in the simulations. So, a good agreement between experiment and simulation is observed.

The simulated events have been constrained as the experimental data and, remarkably, the range of the resulting simulated impact parameters, i.e., about $2 \leq \mathrm{b} \leq 8 \mathrm{fm}$, was found to be consistent with the evaluated experimental one, as deduced by using the Cavata method [25].
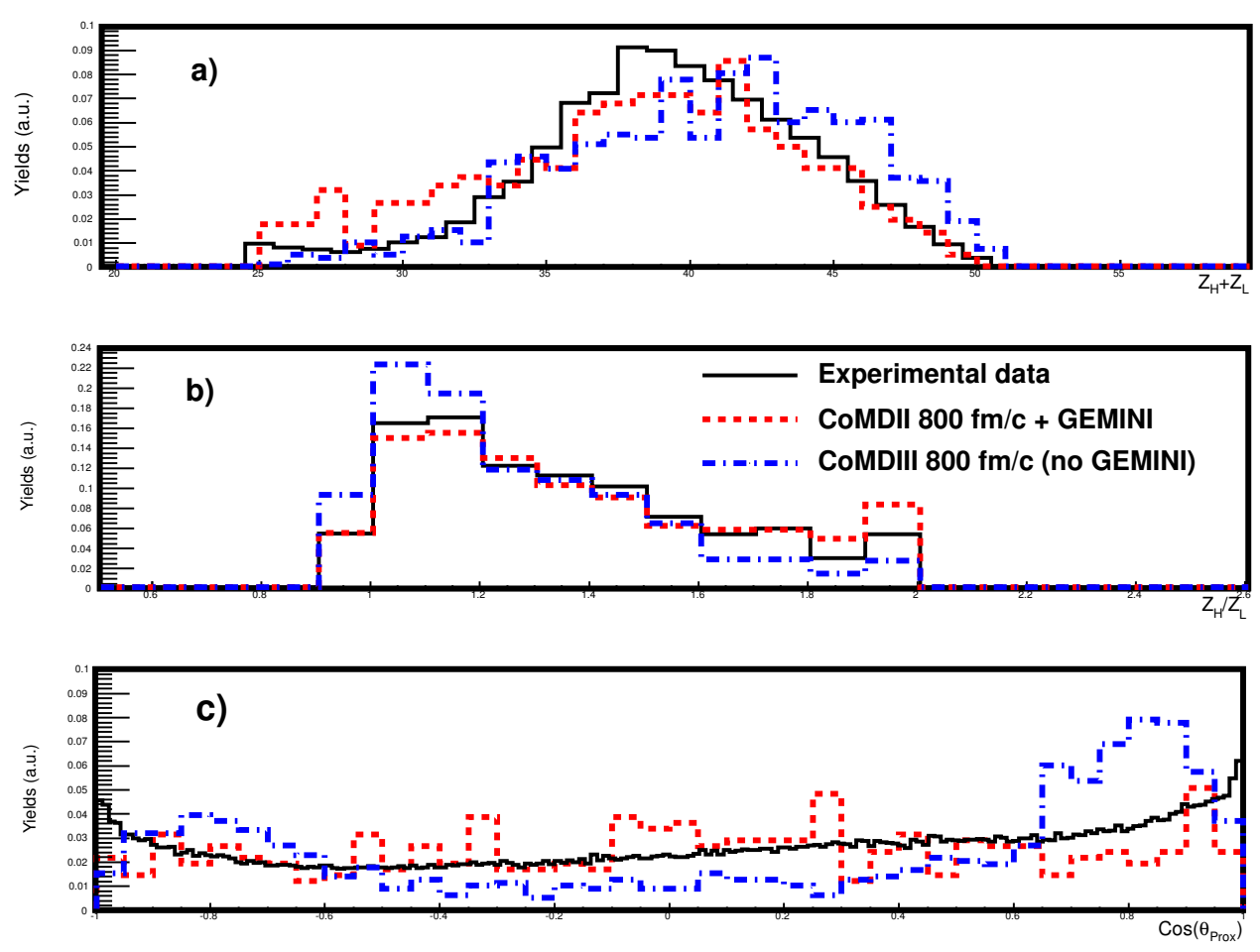

Figure 6: Comparisons between simulated and experimental observables (see text).

In Fig. 7 the comparison between the IMF-IMF experimental correlation functions (in the figure the three ranges of $Z_{A s y}$, like in Fig. 1, are reported for simplicity) and the simulated ones for the largest range of the charge sum, $25 \leq Z_{H}+Z_{L} \leq 50$ is shown. Full squares (in red ) have been evaluated in the case of stopping time of $800 \mathrm{fm} / \mathrm{c}$ in CoMDIII simulations and the full dots (in green) in the case of a shorter time computation of $650 \mathrm{fm} / \mathrm{c}$. This comparison was done by using only the dynamical computation taken at the end of the evolution time in the calculation, without taking into account for the possible statistical de-excitation of the primay fragments (as in Fig. 6). The computations are time consuming, and, within the limitation of the present accuracy, a reasonable good agreement between data and simulations is observed for the intermediate and largest asymmetries, indicating that, according to the transport simulations, the case of dy- 
namical breakup/fission occurring within $800 \mathrm{fm} / \mathrm{c}$ is dominated by on asymmetric splitting with intermediate or large charge asymmetries.

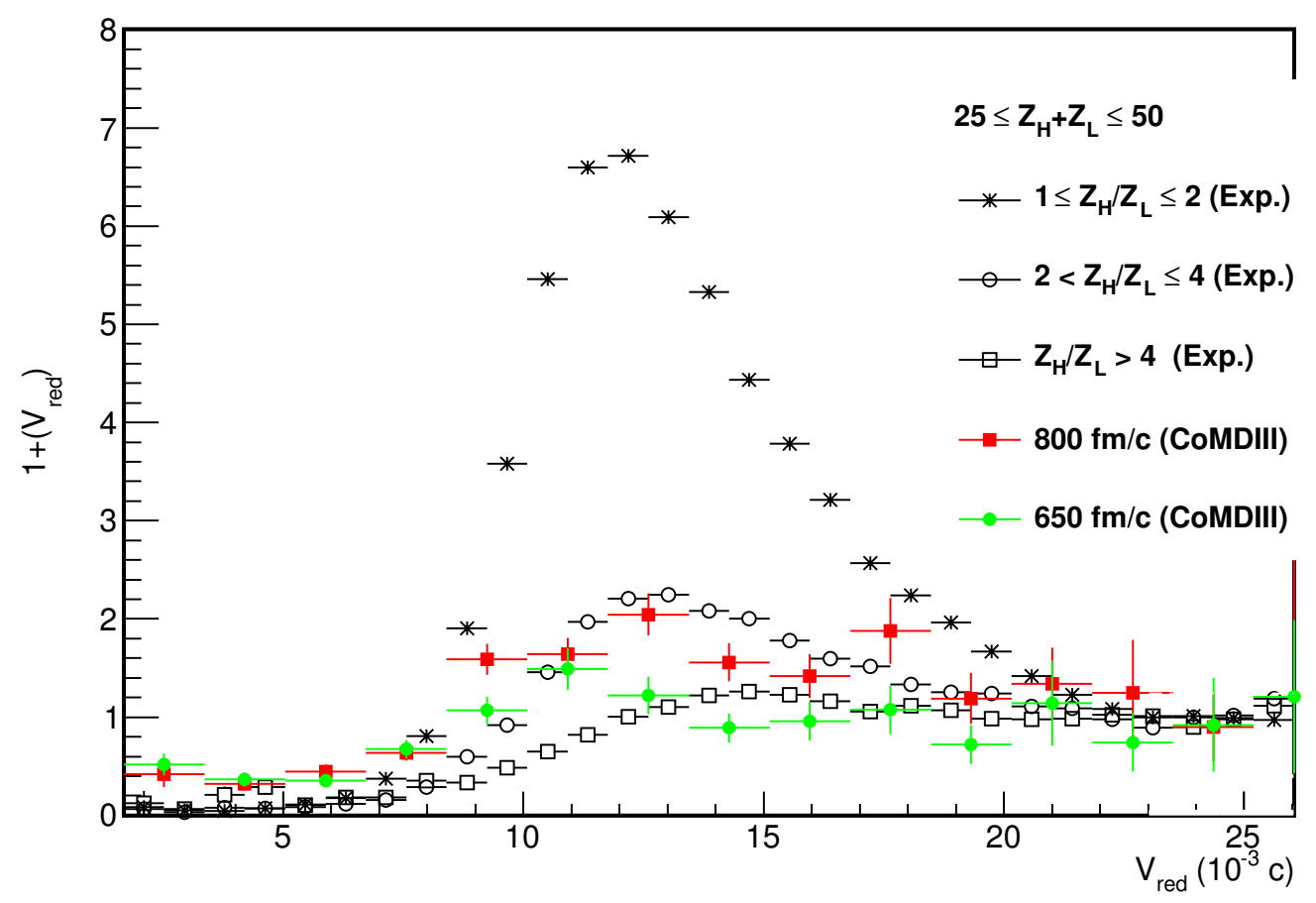

Figure 7: Comparisons between theoretical and experimental correlation functions.

However, it is also envisaged to continue the comparison using the CoMD and also with other transport models in order to obtain information on the reaction mechanism and in medium effective interaction that are as much as possible "model independent". For instance, dynamical fission processes have been investigated also in the context of stochastic mean field models, such as SMF and BLOB [26,27].

\section{Conclusions and future perspectives}

In this work a first detailed study of the IMF-IMF correlation functions of particle pairs of both high relative momenta and charges, in the specific case of dynamical PLF fission in the collision ${ }^{124} \mathrm{Sn}+{ }^{64} \mathrm{Ni}$ at $35 \mathrm{AMeV}$ [13] has been presented. The goal of this study was to demonstrate that the correlation function for massive fragments (strength and shape) is a powerful and complementary experimental method to gain information on the time scale, size and dissipative mechanism of multiple massive IMF emission (this analysis is limited to the value of IMFs multiplicity equal to two) in nuclear reactions at Fermi energies. Important sources of information are expected to be available by careful comparisons of experimental data with advanced nuclear dynamics simulations. The results discussed in this paper strongly encourage for further detailed investigations with new exclusive experiments in this field [28]. 


\section{References}

[1] Henzl V. et al., Phys. Rev. C, 85 (2012) 014606.

[2] Koonin S. E., Phys. Lett. B, 70 (1977) 43.

[3] Pagano E. V., Nuovo Cimento C, 36 (issue 4) (2013) 9.

[4] Pagano E.V. et al., Il Nuovo Cimento C, 39 (2016) 404.

[5] Pagano E.V, PhdThesis, Univerity of Catania, A. A. 2016/2017.

[6] Kim Y. D. et al., Phys. Rev. C, 45 (1992) 338.

[7] Schapiro O., DeAngelis A. R. and Gross D. H. E., Nucl. Phys. A, 568 (1994) 333.

[8] Pal S., Nucl. Phys. A, 594 (1995) 156.

[9] Z. G. Xiao et al., Phys. Lett. B 639 (2006) 436-440.

[10] Geraci E. et al., Nucl. Phys. A, 732 (2004) 173.

[11] Maiolino C. et al., Proceedings of the XLIII International Winter Meeting on Nuclear Physics, Bormio, edited by Iori I. and Bortolotti A., Vol. 124, (2005) p. 194.

[12] De Filippo E. et al., Phys. Rev. C, 71 (2005) 064604.

[13] Russotto P. et al., Phys. Rev. C, 81 (2010) 064605.

[14] G. I. Kopylov and Podgoretsky, Sov. J. Nucl. Phys. 15, 219 (1972).

[15] R. Lednicky and V.L Lyuboshitz, Sov. J. Nucl. Phys. 35, 770 (1982).

[16] R. Lednicky et al., Phys. Lett B 373, 30 (1996).

[17] M. Papa, T. Maruyama and A. Bonasera, Phys. Rev. C, 64 (2001) 024612.

[18] M. Papa, PHYSICAL REVIEW C 87, 014001 (2013).

[19] Lisa M. A., Gong W. G., Gelbke C. K. and Lynch W. G., Phys. Rev. C, 44 (1991) 2865.

[20] Chajecki Z. and Lisa M. A., PRC 78, 064903, (2008).

[21] Chajecki Z. and Lisa M.A., PRC 79, 034908, (2009).

[22] Pratt S. and Ztang B. PRC 36, 6 (1987).

[23] Viola V. E., Kwiatkowski K. and Walker M., Phys. Rev. C, 31 (1985) 1550.

[24] De Filippo E. et al., PRC 86, 014610 (2012).

[25] De Filippo E. and Pagano A., Eur. Phys. J. A, 50 (2014) 32.

[26] C.Rizzo et al., PHYSICAL REVIEW C 90, 054618 (2014).

[27] P.Napolitani, M.Colonna, EPJ Web of Conferences 117, 07008 (2016).

[28] E.Defilippo, E.V.Pagano, P.Russotto et al. CHIFAR @LNS, approved proposal. 